P-242 JUNIOR DOCTORS CARING FOR THE DYING: A PILOT OF PEER-PEER TEACHING IMPROVES CONFIDENCE IN ENDOF-LIFE CARE

${ }^{1,2}$ Simon Haslam, ${ }^{1,2}$ Alice King, ${ }^{1,2}$ Caroline Pinckney, ${ }^{1,2}$ Rameshwor Sunar, ${ }^{1,3}$ Becky Baines. ${ }^{1}$ Royal Devon and Exeter NHS Foundation Trust, Exeter, UK; ${ }^{2}$ Joint first authors; ${ }^{3}$ Hospiscare, Exeter

\subsection{6/bmjspcare-2016-001245.263}

Background The National Care of the Dying Audits have described a need for improvements in end-of-life care in hospitals and highlighted the need for further training for doctors of all levels of seniority. Junior doctors (JDs) are often at the front line of end-of-life care and are well placed to address issues.

Aims

- To identify the challenges for JDs when they are providing end of life care in hospitals.

- To address these through training and quality improvement methodology.

Methods

- Electronic questionnaire to all Foundation Years (FY) JDs working within one medium sized UK teaching hospital.

- Peer-to-peer teaching on core topics including use of casebased discussions.

- Simple rating of confidence in managing patients at the end of their lives on 10-point scale, before and after sessions.

- Mentoring for the peer educators from a palliative care consultant.

Results 21 FY Doctors responded to an electronic questionnaire. Their main needs for education were: help with recognising the dying patient, symptom control, nutrition and hydration issues, and communicating management decisions at the end-of-life.

Two lunchtime sessions were delivered to cover these issues. Attendance was on a voluntary basis. Mixed teaching methods were used: short presentations on key topics and case based discussions. A total of 15 JDs attended one or both sessions.

Mean self-rated scores of confidence in managing patients at the end of life improved:

- 2.4 points for those attending 2 sessions $(n=5)$

- 3 points for those attending 1 session $(n=9)$

Further training in communication skills was highlighted as key by many JDs.

Conclusion Short mixed methods lunch time sessions attracted good voluntary attendance and improved confidence in JDs caring for those at the end of life. Practical tips - both for prescribing and communication were highly valued. Further lunchtime communication skills sessions are planned.

\section{P-243 MEETING THE PHYSICAL ASSESSMENT SKILLS (PAS) NEEDS OF SPECIALIST PALLIATIVE CARE NURSES}

Charlotte Williams, Dominic Whitehouse. St Wilfrid's Hospice, Chichester, UK

\subsection{6/bmjspcare-2016-001245.264}

Clinical nurse specialists (CNS) in palliative care have been encouraged to extend their role. Many see these developments as an opportunity to improve the care of their patients and the therapeutic relationship. Courses in physical assessment skills often extend learning beyond the specialist needs of a hospice CNS. It was our experience that learning was then diluted and failed to meet more specific needs in the context of palliative care.

In response, St Wilfrid's Hospice developed a three-day focused physical assessment skills course for the hospice CNS, delivered to two groups of six students. During the three days participants were provided with a comprehensive course booklet and participated in a mixture of didactic and practical skills sessions.

Topics focused on clinical assessment of the chest, abdomen, cardiovascular and neurological systems, a painful leg, and mental health assessment; with particular reference to case scenarios commonly seen among hospice patients. 100\% of the students rated the course as excellent for structure, content, relevance and application. All agreed they would recommend this course to others. On a scale of $0-10$ (10 being fully competent and 0 Clueless) students rated their skills prior to the course and on completion as follows

\begin{tabular}{lll}
\hline & Pre course range & Post course range \\
\hline General confidence in PAS & $1-3 / 10$ & $6-7 / 10$ \\
General knowledge in PAS & $1-4 / 10$ & $6-8 / 10$ \\
Examination of abdomen with distension & $1-4 / 10$ & $8-10 / 10$ \\
Examination of breathless patient & $1-3 / 10$ & $6-10 / 10$ \\
Mental health assessment & $1-4 / 10$ & $5-10 / 10$ \\
Examination of suspected cord compression & $1-3 / 10$ & $8-10 / 10$ \\
\hline
\end{tabular}

Students who completed the course went on to use their skills in their daily practice and have provided verbal feedback on the positive impact it has had on their patient care and relationships with medical colleagues. We would like to be able to offer this model of specialist palliative care physical assessment skills training to other hospices.

\section{P-244 SPRINGHILL HOSPICE PALLIATIVE CARE EDUCATION PASSPORT}

Jane Ashworth. Springhill Hospice, Rochdale, UK

\subsection{6/bmjspcare-2016-001245.265}

Background An innovative programme designed by the author to meet the needs of community care staff in the borough, the PCEP accredits the care worker rather than the organisation. High staff turnover is prevalent in care homes and the aim is that all care homes in the borough will have at least $70 \%$ of staff trained by 2019 .

Methods The PCEP is based on the Common Core Competencies and Principles for Health and Social Care Workers (2014), mapped against Recommended Core Education Standards for Care and Support for the Dying Person in the Last Days and Hours of Life (SCN 2014), and incorporates many aspects of the National Care Certificate (2015). Skills are transferrable, and once part of everyday practice will enhance the care of all service users.

The SPCP comprises of six core modules:-

- Northwest EoL care model, difficult decisions and recognising advanced disease

- Communication skills

- Spirituality/psychological needs and supporting families and carers 